https://doi.org/10.15407/ujpe65.11.987

A. MAIRECHE

Laboratory of Physics and Material Chemistry, Physics department, Sciences Faculty, University of M'sila (BP 239 Chebilia-M'sila, Algerai; e-mail: abdelmadjid.maireche@univ-msila.dz)

\title{
A NEW APPROACH TO THE APPROXIMATE ANALYTIC SOLUTION OF THE THREE-DIMENSIONAL SCHR'ODINGER EQUATION FOR HYDROGENIC AND NEUTRAL ATOMS IN THE GENERALIZED HELLMANN POTENTIAL MODEL
}

\begin{abstract}
Within the framework of nonrelativistic noncommutative quantum mechanics using the improved approximation scheme to the centrifugal term for any l-states via the generalized Bopp's shift method and standard perturbation theory, we have obtained the energy eigenvalues of a newly proposed generalized Hellmann potential model (the GHP model) for the hydrogenic atoms and neutral atoms. The potential is a superposition of the attractive Coulomb potential plus Yukawa one, and new central terms appear as a result of the effects of noncommutativity properties of space and phase in the Hellmann potential model. The obtained energy eigenvalues appear as a function of the generalized gamma function, the discrete atomic quantum numbers $(j, n, l, s$ and $m)$, infinitesimal parameters $(a, b, \delta)$ which are induced by the positionposition and phase-phase noncommutativity, and, the dimensional parameters $(\Theta, \bar{\theta})$ of the GHP model, in the nonrelativistic noncommutative three-dimensional real space phase (NC: 3D-RSP). Furthermore, we have shown that the corresponding Hamiltonian operator with (NC: $3 D-R S P)$ symmetries is the sum of the Hamiltonian operator of the Hellmann potential model and two operators, the first one is the modified spin-orbit interaction, while the second is the modified Zeeman operator for the hydrogenic and neutral atoms.
\end{abstract}

Keywords: Schrödinger equation, Hellmann potential model, noncommutative quantum mechanics, star product, generalized Bopp's shift method.

\section{Introduction}

Over the past years, the Hellmann potential model, which is a superposition of the attractive Coulomb potential and a Yukawa potential, has received much concern from many authors. By applying an alternative perturbative scheme in solving the Schrödinger equation (SE) for the Yukawa potential model with a modified screening parameter S.M. Ikhdair and R. Sever investigated energy levels of neutral atoms

(C) A. MAIRECHE, 2020

ISSN 2071-0194. Ukr. J. Phys. 2020. Vol. 65, No. 11
[1]. Furthermore, various authors to represent the electron-core or the electron-ion interaction have used this potential. Hellmenn was the first who suggested this potential, and it was studied in both the relativistic and nonrelativistic quantum mechanics (see, e.g., [2-7]). In particular, it is used to describe the interactions of hydrogenic atoms. This physical potential has been the attraction and interest for many researchers; we mention G. Kocak et al., M. Hamzavi et al., S.M. Ikhdair et al., and C.A. Onate et al. They solved the SE in their works with the Hellmann po- 
tential model using several methods such as the parametric $\mathrm{N}-\mathrm{U}$ method, asymptotic iteration, and the supersymmetric approach (see, e.g., [4-7]). It should be noted that C.O. Edet et al. were the first who generalize this Hellmann-Kratzer potential model to include the Kratzer-like potential $V(r)=D_{e}\left(\frac{r-r_{e}}{r_{e}}\right)$ [8]. H. Louis et al. solved the Dirac equation for the Manning-Rosen plus shifted Deng-Fan potential and the Coulomb-like tensor interaction using the $\mathrm{N}-\mathrm{U}$ method [9]. Hitler et al. studied the Klein-Gordon equation for the more general exponential screened Coulomb potential plus Yukawa potential using the $\mathrm{N}-\mathrm{U}$ method and obtained the relativistic and nonrelativistic wave equations for modified HylleraasHulthen potential using the $\mathrm{N}-\mathrm{U}$ quantum formalism $[10,11]$. C.O. Edet et al. obtained analytic solutions of the SE with non-central generalized inverse quadratic Yukawa potential and $q$-deformed Hulthen plus generalized inverse quadratic Yukawa potential in arbitrary dimensions $[12,13]$. U.S. Okorie et al. solved the Klein-Gordon equation (KGE) with a generalized hyperbolic potential in D-dimensions [14]. B.I. Ita et al. obtained the approximate solution to the SE with Manning-Rosen plus a class of Yukawa potential via WKBJ approximation [15]. Furthermore, C.O. Edet et al. studied bound-state solutions of the Schrödinger equation for the modified Kratzer potential plus screened Coulomb potential [16]. In this work, motivated by several recent studies such as the non-renormalizable of the standard model, string theory, quantum gravity, the noncommutative quantum mechanics (NCQM) has attracted much attention (see, e.g., [17-23]). Recently, we are studied the KGE with modified Coulomb potential plus inverse-square-root potential and modified Coulomb plus inverse-square potential in the noncommutative three-dimensional space $[18,19]$. The noncommutativity of space-phase was known first by Heisenberg in 1930 and was formalized by Snyder in 1947. In the present research paper, we want to extend, the study in Refs. [4-8] to the case of nonrelativistic NCQM to the possibility of finding other applications and more profound interpretations in the sub-atomic scales. The nonrelativistic energy levels for the hydrogenic atoms and neutral atoms such as sodium, carbon, and gold described by the generalized Hellmann potential model (GHP model) in the context of a noncommutative space phase, have not been obtained yet. The main purpose of this paper is to solve the modified Schrödinger equation (MSE) with the GHP model (see below):

$V_{h p}(r)=-\frac{a}{r}+\frac{b \exp (-\delta r)}{r} \rightarrow$

$\rightarrow V_{n c}^{h p}(r)=V_{h p}(r)+$

$+\left(\frac{b \exp (-\delta r)}{2 r^{3}}+\frac{b \delta \exp (-\delta r)}{2 r^{2}}-\frac{a}{2 r^{3}}\right) \mathbf{L} \boldsymbol{\Theta}$

in (NC: 3D-RSP) symmetries using the generalized Bopp's shift method which depends on the concepts that we will present below in the third section. It is worth to mention that the Hellmann-Kratzer potential (Eq. (1)) used in the present study was first proposed by Edet et al. [8]. The structure of nonrelativistic noncommutative quantum mechanics (NRNCQM) based to $\mathrm{NC}$ canonical commutations relations in the Schrödinger, Heisenberg, and interaction pictures (SP, HP, and IP), respectively, as follows (throughout this paper, the natural units $c=\hbar=1$ will be used) (see, e.g., [20-27]):

$$
\begin{aligned}
& {\left[\hat{x}_{i} * \hat{p}_{j}\right]=\left[\hat{x}_{i}(t) * * \hat{p}_{j}(t)\right]=\left[\hat{x}_{I i}(t) * \hat{p}_{I j}(t)\right]=i \delta_{i j}} \\
& {\left[\hat{x}_{i} * \hat{x}_{j}\right]=\left[\hat{x}_{i}(t) * * \hat{x}_{j}(t)\right]=\left[\hat{x}_{I i}(t)^{*}, \hat{x}_{I j}(t)\right]=i \theta_{i j}} \\
& {\left[\hat{p}_{i}^{*}, \hat{p}_{j}\right]=\left[\hat{p}_{i}(t) * \hat{p}_{j}(t)\right]=\left[\hat{p}_{I i}(t) * * \hat{p}_{I i}(t)\right]=i \bar{\theta}_{i j}}
\end{aligned}
$$

However, the operators $\widehat{\zeta}_{i H}(t)=\left(\hat{x}_{i} \vee \hat{p}_{i}\right)(t)$ and $\widehat{\zeta}_{i I}(t)=\left(\hat{x}_{i I} \vee \hat{p}_{i I}\right)(t)$ (in HP and IP, respectively) depend on the corresponding operator $\widehat{\zeta}_{i s}=\hat{x}_{i} \vee \hat{p}_{i}$ in SP from the following projections relations:

$$
\begin{gathered}
\left\{\begin{array}{l}
\zeta_{i H}(t)=\exp \left(i \widehat{H}_{h p} T\right) \zeta_{i s} \exp \left(-i \widehat{H}_{h p} T\right) \\
\zeta_{i I}(t)=\exp \left(i \widehat{H}_{o h p}\left(t-t_{0}\right)\right) \zeta_{i s}(t) \exp \left(-i \widehat{H}_{o h p} T\right)
\end{array} \Rightarrow\right. \\
\Rightarrow\left\{\begin{array}{l}
\widehat{\zeta}_{i H}(t)=\exp \left(i \widehat{H}_{n c}^{h p} T\right) \widehat{\zeta}_{i s} \exp \left(-i \widehat{H}_{n c}^{h p} T\right) \\
\widehat{\zeta}_{i I}(t)=\exp \left(i \widehat{H}_{o n c}^{h p} T\right) \widehat{\zeta}_{i s} \exp \left(-i \widehat{H}_{o n c}^{h p} T\right) .
\end{array}\right.
\end{gathered}
$$

Here, $T=t-t_{0}, \zeta_{i s}=x_{i} \vee p_{i}, \widehat{\zeta}_{i H}(t)=\left(x_{i} \vee p_{i}\right)(t)$ and $\widehat{\zeta}_{i I}(t)=\left(\hat{x}_{i I} \vee \hat{p}_{i I}\right)(t)$ are the three representations (SP, HP, and IP) in NRQM, while the dynamics of new systems $\frac{d \widehat{\zeta}_{i H}(t)}{d t}$ is described by the following motion equations in NRNCQM:

$$
\frac{d \zeta_{i H}(t)}{d t}=\left[\zeta_{i H}(t), \widehat{H}_{h p}\right]+\frac{\partial \zeta_{i H}(t)}{\partial t} \Rightarrow
$$

ISSN 2071-0194. Ukr. J. Phys. 2020. Vol. 65, No. 11 
$\Rightarrow \frac{d \widehat{\zeta}_{i H}(t)}{d t}=\left[\widehat{\zeta}_{i H}(t) * \widehat{H}_{n c}^{h p}\right]+\frac{\partial \widehat{\zeta}_{i H}(t)}{\partial t}$

Here, $\widehat{H}_{h p}\left(\widehat{H}_{o h p}\right)$ and $\widehat{H}_{n c}^{h p}\left(\widehat{H}_{o n c}^{h p}\right)$ denote the ordinary and generalized quantum Hamiltonian (free) operators for the GHP model in the NRQM and NRNCQM, respectively. The very small two parameters $\left(\theta^{i j}, \bar{\theta}^{i j}\right)=\epsilon^{i j}(\theta, \bar{\theta})$ (compared to the energy) are elements of two antisymmetric real matrices with dimensions of (length) ${ }^{2}$ and (momentum) ${ }^{2}$, respectively. Furthermore, $(*)$, denotes the star product, which is generalized for two arbitrary functions $(f, g)(x, p)$ to the form $(\widehat{f}, \widehat{g})(\widehat{x}, \widehat{p})$ in the ordinary $3-$ dimensional space-phase (see, e.g., [25-33]):

$(f * g)(x, p)=$

$=\left(f g-\frac{i}{2} \theta^{i j} \frac{\partial f}{\partial x^{i}} \frac{\partial g}{\partial x^{j}}-\frac{i}{2} \bar{\theta}^{i j} \frac{\partial f}{\partial p^{i}} \frac{\partial g}{\partial p^{j}}\right)(x, p)$.

This allows the formation of a scale of two space and phase cells with volumes $l_{n c s}^{3}=\theta^{3 / 2}$ and $l_{n c p}^{3}=$ $=\bar{\theta}^{3 / 2}$, respectively. On the other hand, Eq. (5) allows us to satisfy the postulated algebra in Eq. (2). The second and the third terms in the above equation are the effects of (space-space) and (phase-phase) noncommutativity properties, respectively. It is the aim of this paper to present approximate solutions of the modified Schrödinger equation with the GHP model in 3-dimensions using the improved approximation scheme to the centrifugal term for different $l$ zero states and generalized Bopp's shift method, in addition to the standard perturbation theory. The organization scheme of the recent work is given as follows: In the next section, we briefly review the ordinary SE with the HP model based on Refs. [38]. Section 3 is devoted to studying the MSE by applying the generalized Bopp's shift method for the GHP model. In the next subsection, by applying standard perturbation theory to find the quantum spectrum of $n^{t h}$ excited levels for the spin-orbital interaction in the framework of the global group (NC-3D: RSP). Then we derive the magnetic spectrum for the GHP model. In the fourth section, we resume the global spectrum and corresponding NC Hamiltonian operator for the GHP model and corresponding energy levels of the hydrogenic atoms such as $\mathrm{He}^{+}$, $\mathrm{Li}^{+2}$, and $\mathrm{Be}^{+}$and, in addition, the neutral atoms such as sodium, carbon, and gold atoms. Finally, the achieved results are briefly summarized in the last section.

\section{Overview of the Eigenfunctions and the Energy Eigenvalues for Hellmann Potential Model in NRQM}

In this section, we recall the time-independent SE for the Hellmann potential model, which is an important short-range potential that behaves itself like the Coulomb potential for small values of the distance between two particles $r$ and decreases exponentially for its large values (see, e.g., $[3-8]$ ):

$V_{h p}(r)=-\frac{a}{r}+\frac{b \exp (-\delta r)}{r}$,

where $a, b$, and $\delta$ are the potential strengths of (Coulomb and Yukawa) potentials and the screening parameter, respectively. If we insert this potential into the $\mathrm{SE}$, we obtain the radial part of the $\mathrm{SE}$ as follows:

$\frac{d^{2} U_{n l}(r)}{d r^{2}}+\frac{2}{r} \frac{d U_{n l}(r)}{d r}+$

$+2 \mu\left(E_{n l}-V_{-h p}(r)\right) U_{n l}(r)=0$

and

$\frac{d^{2} R_{n l}(r)}{d r^{2}}+2 \mu\left(E_{n l}-V_{\text {eff }-h p}(r)\right) R_{n l}(r)=0$.

Here, $U_{n l}(r)=\frac{R_{n l}(r)}{r}$ and $V_{\text {eff }-h p}(r)=V_{h p}(r)+\frac{l(l+1)}{r^{2}}$ is the effective potential, $\mu$ is the reduced mass, $E_{n l}$ is the eigenvalues of the Hellmann potential model, while $n$ and $l$ are the radial and orbital angular momentum quantum numbers. The complete wave function $\Psi(r, \theta, \varphi)=U_{n l}(r) Y_{l}^{m}(\theta, \varphi)$, as a function of the Jacobi polynomial $P_{n}^{\left(2 \eta_{n}, 2 l+1\right)}(1-2 s)$ and the spherical harmonic functions $Y_{l}^{m}(\theta, \varphi)$, is as follows (see, e.g., $[4-7])$ :

$\Psi(r, \theta, \varphi)=N_{n l} \frac{s^{-\delta \eta_{n}}}{r}(1-s)^{l+1} P_{n}^{\left(2 \eta_{n}, 2 l+1\right)}(1-2 s) \times$ $\times Y_{l}^{m}(\theta, \varphi)$.

Here, $s=\exp (-\delta r), \eta_{n}=\sqrt{\frac{\epsilon_{n}-A}{\delta^{2}}+l(l+1)}, \epsilon_{n}=$ $=-2 \mu E_{n l}, A=2 \mu a \delta, N_{n l}=\sqrt{\frac{n \delta a \Gamma(n+a+\nu+1) l}{\Gamma(n+a+1) \Gamma(n+\nu+1)}}$ is the normalization constant and $\nu=2 l+1$. The energy $E_{n l}$ of the potential in Eq. (6) is given by (see, e.g., $[4-6]$ ):

$$
\begin{aligned}
& E_{n l}=-\frac{\delta^{2}}{2 \mu}\left[\left(\frac{(2 \mu / a)(a-b)-(n+l+1)^{2}-l(l+1)}{2(n+l+1)}\right)^{2}+\right. \\
& \left.+l(l+1)+\frac{2 \mu a}{\delta}\right]
\end{aligned}
$$




\section{Solution of MSE for GHP Model}

\subsection{Physical model}

In this subsection, we devote this part to studying for the GHP model $V_{n c}^{h p}(r)$, in (NC: 3D-RSP) symmetries. To solve this task, the physical form of a modified Schrödinger equation MSE, it is necessary to replace the ordinary three-dimensional Hamiltonian operators $\widehat{H}_{h p}(p, x)$, ordinary complex wave function $\Psi(\mathbf{r})$ and $E_{n l}$ (in NRQM) by threedimensional Hamiltonian operators $\widehat{H}_{n c}^{h p}(\widehat{p}, \widehat{x})$, complex wave function $\Psi(\widehat{\mathbf{r}})$, and new values of $E_{n c}^{h p}$, respectively (in NRNCQM). In addition, to replace the ordinary product by the star product $(*)$. This allows us to construct the MSE in (NC-3D: RSP) symmetries as (see, e.g., [34-38]):

$\widehat{H}_{h p}(p, x) \Psi(\mathbf{r})=E_{n l} \Psi(\mathbf{r}) \Rightarrow$

$\Rightarrow \widehat{H}_{n c}^{h p}(\widehat{p}, \widehat{x}) * \Psi(\widehat{\mathbf{r}})=E_{n c}^{h p} \Psi(\widehat{\mathbf{r}})$.

The Bopp's shift method employed in the solutions enables us to explore an effective way of obtaining the modified potential in NRNCQM. It is based on the following commutators (see, e.g., [22-27]):

$$
\begin{gathered}
{\left[\hat{x}_{i}, \hat{x}_{j}\right]=\left[\hat{x}_{i}(t) \hat{x}_{j}(t)\right]=\left[\hat{x}_{I i}(t), \hat{x}_{I j}(t)\right]=i \theta_{i j}} \\
{\left[\hat{p}_{i} \hat{p}_{j}\right]=\left[\hat{p}_{i}(t), \hat{p}_{j}(t)\right]=\left[\hat{p}_{I i}(t), \hat{p}_{I i}(t)\right]=i \bar{\theta}_{i j}}
\end{gathered}
$$

The generalized positions and momentum coordinates $\left(\hat{x}_{i}, \hat{p}_{i}\right)$ in (NC: $\left.3 \mathrm{D}-\mathrm{RSP}\right)$ depend on the corresponding usual generalized positions and momentum coordinates $\left(x_{i}, p i\right)$ in NRQM as follows, respectively (see, e.g., [34-38]):

$\left(x_{i}, p i\right) \rightarrow\left(\hat{x}_{i}, \hat{p}_{i}\right)=\left(x_{i}-\frac{\theta_{i j}}{2} p_{j}, p_{i}+\frac{\bar{\theta}_{i j}}{2} x_{j}\right)$.

The above equation allows us to obtain the two operators $\hat{r}^{2}$ and $\hat{p}^{2}$ in (NC-3D: RSP) (see, e.g., [36-42]):

$$
\left(r^{2}, p^{2}\right) \rightarrow\left(\hat{r}^{2}, \hat{p}^{2}\right)=\left(r^{2}-\mathbf{L \Theta}, p^{2}+\mathbf{L} \overline{\boldsymbol{\theta}}\right) .
$$

The two couplings $\mathbf{L} \Theta$ and $\mathbf{L} \overline{\boldsymbol{\theta}}$ are $\left(L_{x} \Theta_{12}+\right.$ $\left.+L_{y} \Theta_{23}+L_{z} \Theta_{13}\right)$ and $\left(L_{x} \bar{\theta}_{12}+L_{y} \bar{\theta}_{23}+L_{z} \bar{\theta}_{13}\right)$, respectively, and $\left(L_{x}, L_{y}\right.$ and $\left.L_{z}\right)$ are the three components of the angular momentum operator, while $\Theta_{i j}==\theta_{i j /} / 2$. Thus, the reduced Schrödinger equation (without star product) can be written as:

$\widehat{H}_{n c}^{h p}(\widehat{p}, \widehat{x}) * \Psi(\widehat{\mathbf{r}})=E_{n c}^{h p} \Psi(\widehat{\mathbf{r}}) \Rightarrow$
$\Rightarrow H_{n c}^{h p}(\widehat{p}, \widehat{x}) \Psi(\mathbf{r})=E_{n c}^{h p} \Psi(\mathbf{r})$.

The Hamiltonian operator $H_{n c}^{h p}(\widehat{p}, \widehat{x})$ for the GHP model can be expressed as:

$H_{n c}^{h p}(\widehat{p}, \widehat{x})=H\left(\widehat{x}_{i}=x_{i}-\frac{\theta_{i j}}{2} p_{j}, \widehat{p}_{i}=p_{i}+\frac{\bar{\theta}_{i j}}{2} x_{j}\right)$.

Now, we want to find the GHP model for $V_{n c}^{h p}(r)$ in (NC-3D: RSP) symmetries:

$V_{h p}(r) \rightarrow V_{n c}^{h p}(\widehat{r})=-\frac{a}{\widehat{r}}+\frac{b \exp (-\delta \widehat{r})}{\widehat{r}}$.

After straightforward calculations, we can obtain the important terms $\left(-\frac{a}{\widehat{r}}\right)$ and $\left(\frac{b \exp (-\delta \widehat{r})}{\widehat{r}}\right)$ which will be used to determine the GHP model in (NC: 3DRSP) symmetries as:

$\left\{\begin{array}{l}-\frac{a}{\widehat{r}}=-\frac{a}{r}-\frac{a}{2 r^{3}} \mathbf{L} \Theta+O\left(\Theta^{2}\right), \\ b \exp (-\delta \widehat{r})=b \exp (-\delta r)+\frac{\delta b}{2 r} \exp (-\delta r) \mathbf{L} \Theta+O\left(\Theta^{2}\right) .\end{array}\right.$

This gives

$\frac{b \exp (-\delta \widehat{r})}{\widehat{r}}=\frac{b \exp (-\delta r)}{r}+\frac{b}{2 r^{3}} \exp (-\delta r) \mathbf{L} \boldsymbol{\Theta}+$

$+\frac{\delta b}{2 r^{2}} \exp (-\delta r) \mathbf{L} \Theta+O\left(\Theta^{2}\right)$.

By making the substitution of the above equations (18) and (17) into Eq. (15), we find our global working Hamiltonian operator $H_{n c}^{h p}(\widehat{p}, \widehat{x})$ that satisfies the equation in (NC: 3D-RSP) symmetries:

$H_{n c}^{h p}(\widehat{p}, \widehat{x})=\left(\frac{b \exp (-\delta r)}{2 r^{3}}+\frac{\delta b \exp (-\delta r)}{2 r^{2}}-\frac{a}{2 r^{3}}\right) \mathbf{L} \mathbf{\Theta}+$ $+\frac{\mathbf{L} \boldsymbol{\theta}}{2 \mu}+\widehat{H}_{h p}(p, x)$

where the operator $\widehat{H}_{h p}(p, x)$ is just the ordinary Hamiltonian operator in NRQM:

$\widehat{H}_{h p}(p, x)=\frac{p^{2}}{2 \mu}-\frac{a}{r}+\frac{b \exp (-\delta r)}{r}$,

while the rest four terms are proportional with two infinitesimals parameters $(\Theta$ and $\bar{\theta})$. We can considered them as perturbation terms $H_{\text {pert }}^{h p}(\widehat{p}, \widehat{x})$ in $(\mathrm{NC}$ : 3D-RSP) symmetries as:

$H_{\text {pert }}^{h p}(\widehat{p}, \widehat{x})=$

ISSN 2071-0194. Ukr. J. Phys. 2020. Vol. 65, No. 11 
$=\left(\frac{b \exp (-\delta r)}{2 r^{3}}+\frac{\delta b \exp (-\delta r)}{2 r^{2}}-\frac{a}{2 r^{3}}\right) \mathbf{L} \boldsymbol{\Theta}+\frac{\mathbf{L}}{\overline{\boldsymbol{\theta}}} 2 \mu$.

It is clear that the operator $\widehat{H}_{h p}(p, x)$ is just the Hamiltonian operator for hydrogenic atoms such as $\mathrm{He}^{+}, \mathrm{Li}^{+2}$ and $\mathrm{Be}^{+}$in ordinary quantum mechanics, while the generated part $H_{\text {pert }}^{h p}(\widehat{p}, \widehat{x})$ appears as results of a deformation of the noncommutativity space phase. In the present work, we can disregard the second term in $H_{\text {pert }}^{h p}(\widehat{p}, \widehat{x})$ because we are interested in the corrections of first order $\Theta$ and $\bar{\theta}$.

\subsection{Spin-orbit Hamiltonian operator for the hydrogenic atoms in the GHP model}

In this subsection, we want to derive the physical form of the induced Hamiltonian $H_{\text {pert }}^{h p}(\widehat{p}, \widehat{x})$ due to the space-phase noncommutativity. To achieve this goal, we replace $\mathbf{L} \boldsymbol{\Theta}$ both and $\mathbf{L} \overline{\boldsymbol{\theta}}$ by useful physical forms $\epsilon \Theta \mathbf{L S}$ and $\epsilon \bar{\theta} \mathbf{L S}$, respectively (see, e.g., [39-43]):

$H_{s o}^{h p}(\widehat{p}, \widehat{x})=$

$=\epsilon\left[\left(\frac{b \exp (-\delta r)}{2 r^{3}}+\frac{\delta b \exp (-\delta r)}{2 r^{2}}-\frac{a}{2 r^{3}}\right) \Theta+\frac{\bar{\theta}}{2 \mu}\right] \mathbf{L S}$

Here, $\Theta=\left(\Theta_{12}^{2}+\Theta_{23}^{2}+\Theta_{13}^{2}\right)^{1 / 2}, \bar{\theta}=\left(\bar{\theta}_{12}^{2}+\bar{\theta}_{23}^{2}+\right.$ $\left.+\bar{\theta}_{13}^{2}\right)^{1 / 2}$, and $\epsilon \approx 1 / 137$ is the atomic fine structure constant, and $\mathbf{S}$ denotes the spin of the hydrogenic atoms such as $\left(\mathrm{He}^{+}, \mathrm{Li}^{+2}\right.$, and $\left.\mathrm{Be}^{+}\right)$. Thus, the spinorbit interactions $H_{s o}^{h p}(\widehat{p}, \widehat{x})$ appear automatically as a result of the deformation of the space phase. Now, physically, we can rewrite the quantum spin-orbit LS coupling as follows:

$\mathbf{J}=\mathbf{L}+\mathbf{S} \Rightarrow 2 \mathbf{L S}=\mathbf{J}^{2}-\mathbf{L}^{2}-\mathbf{S}^{2}$.

Here, $\mathbf{J}$ is the total momentum of the hydrogenic atoms such as $\mathrm{He}^{+}, \mathrm{Li}^{+2}$ and $\mathrm{Be}^{+}$. The substitution of this equation into Eq. (22) yields:

$$
\begin{aligned}
& H_{s o}^{h p}(\widehat{p}, \widehat{x})=\epsilon\left[\left(\frac{b \exp (-\delta r)}{2 r^{3}}+\frac{\delta b \exp (-\delta r)}{2 r^{2}}-\right.\right. \\
& \left.\left.-\frac{a}{2 r^{3}}\right) \Theta+\frac{\bar{\theta}}{2 \mu}\right] G^{2},
\end{aligned}
$$

where $G^{2}=\mathbf{J}^{2}-\mathbf{L}^{2}-\mathbf{S}^{2}$ and $\mathbf{J}=\mathbf{L}+\mathbf{S}$. It is well known that the eigenvalues $j$ of the total operator $\mathbf{J}$ can be obtained from the interval $|l-1 / 2| \leq j \leq$ $\leq|l+1 / 2|$ for hydrogenic atoms. We have an occasion of determining two-sided bounds to the eigenvalues of the operator $\mathbf{J}^{2}-\mathbf{L}^{2}-\mathbf{S}^{2}$ as follows:

$k(j, l, s)=j(j+1)-l(l+1)-s(s+1)=$

$= \begin{cases}k_{-}(j=l-1 / 2, l, s) & \text { for spin_down } \\ k_{+}(j=l+1 / 2, l, s) & \text { for spin_down. }\end{cases}$

A second occasion of determining a diagonal matrix $H_{s o}^{\mathrm{hp}}(\widehat{p}, \widehat{x})$ of order $(3 \times 3)$ with diagonal elements $\left(H_{s o}^{h p}(\widehat{p}, \widehat{x})\right)_{11},\left(H_{s o}^{\mathrm{hp}}(\widehat{p}, \widehat{x})\right)_{22}$ and $\left(H_{s o}^{h p}(\widehat{p}, \widehat{x})\right)_{33}=0$ as:

$$
\begin{aligned}
& \left(H_{s o}^{h p}(\widehat{p}, \widehat{x})\right)_{11}= \\
& =\epsilon k_{+}\left[\left(\frac{b \exp (-\delta r)}{2 r^{3}}+\frac{\delta b \exp (-\delta r)}{2 r^{2}}-\frac{a}{2 r^{3}}\right) \Theta+\frac{\bar{\theta}}{2 \mu}\right]
\end{aligned}
$$

if $j=l+1 / 2$,

$\left(H_{s o}^{h p}(\widehat{p}, \widehat{x})\right)_{22}=$

$=\epsilon k_{-}\left[\left(\frac{b \exp (-\delta r)}{2 r^{3}}+\frac{\delta b \exp (-\delta r)}{2 r^{2}}-\frac{a}{2 r^{3}}\right) \Theta+\frac{\bar{\theta}}{2 \mu}\right]$

if $j=l-1 / 2$.

After the straightforward calculation, the radial functions $R_{n l}(r)$ satisfy the following differential equation in NRNCQ for hydrogenic atoms in the GHP model:

$\frac{d^{2} R_{n l}(r)}{d r^{2}}+2 \mu\left(E_{n c}^{\mathrm{hp}}-V_{n c}^{\mathrm{eff}}(r)\right) R_{n l}(r)=0$

with

$V_{n c}^{\mathrm{eff}}(r)=V_{\mathrm{eff}-\mathrm{hp}}(r)+$

$+\epsilon\left[\left(\frac{b \exp (-\delta r)}{2 r^{3}}+\frac{\delta b \exp (-\delta r)}{2 r^{2}}-\frac{a}{2 r^{3}}\right) \Theta+\frac{\bar{\theta}}{2 \mu}\right] \mathbf{L S}$.

We introduced the generalized effective potential $V_{n c}^{\text {eff }}(r)$ in (NC: 3D-RSP) symmetries. We have seen previously that the induced spin-orbit $H_{s o}^{h p}(\widehat{p}, \widehat{x})$ is infinitesimal compared to the principal Hamiltonian operator $H_{h p}(p, x)$ in NRQM for hydrogenic atoms such as $\mathrm{He}^{+}, \mathrm{Li}^{+2}$, and $\mathrm{Be}^{+}$in the GHP model. This equation cannot be solved analytically for any state, 
because of the centrifugal term and the studied potential itself. Therefore, in the present work, we considered the following approximation type suggested by Greene, Aldrich, and Dong et al. for them $[44,45]$ :

$$
\begin{aligned}
& \frac{1}{r^{2}} \approx \frac{\delta^{2} \exp (-\delta r)}{(1-\exp (-\delta r))^{2}} \Rightarrow \\
& \Rightarrow\left\{\begin{array}{l}
\frac{\exp (-\delta r)}{r^{2}} \approx \frac{\delta^{2} s^{2}}{(1-s)^{2}}, \\
\frac{\exp (-\delta r)}{r^{3}} \approx \frac{\delta^{3} s^{5 / 2}}{(1-s)^{3}}, \\
\frac{1}{r^{3}} \approx \frac{\delta^{3} s^{3 / 2}}{(1-s)^{3}} .
\end{array}\right.
\end{aligned}
$$

This allows us to apply standard perturbation theory to determine the nonrelativistic energy corrections $E_{s o}^{h p}$ of hydrogenic atoms such as $\mathrm{He}^{+}, \mathrm{Li}^{+2}$, and $\mathrm{Be}^{+}$for the first order in two infinitesimal parameters $\Theta$ and $\bar{\theta}$ due to the noncommutativity of spacephase properties. Instead of solving the modifiedradial Schrödinger equation for the effective GHP model $V_{n c}^{\text {eff }}(r)$ given by Eq. $(27 \mathrm{~b})$, we now solve the modified radial Schrödinger equation for the generalized effective potential given by the previous approximations:

$$
\begin{aligned}
& V_{n c}^{\mathrm{eff}}(s)=\epsilon\left[\left(\frac{b \delta^{3} s^{5 / 2}}{(1-s)^{3}}+\frac{\delta^{3} b s^{2}}{(1-s)^{2}}-\frac{a \delta^{3} s^{3 / 2}}{(1-s)^{3}}\right) \Theta+\right. \\
& \left.+\frac{\bar{\theta}}{2 \mu}\right] \mathbf{L S}+\frac{\delta^{2} l(l+1)}{(1-s)^{2}}-\frac{a \delta}{1-s}+\frac{b \delta s}{1-s}
\end{aligned}
$$

\subsection{Bound-state solution \\ for spin-orbit operator for hydrogenic atoms in the GHP model}

The principal goal of this subsection is to determine the energy spectrum $E_{s o}^{u h p}\left(k_{+}, n, a, b, \delta, j, l, s\right) \equiv$ $\equiv E_{s o}^{u h p}$ and $E_{s o}^{d \mathrm{hp}}\left(k_{-}, n, a, b, \delta, j, l, s\right) \equiv E_{s o}^{d h p}$ which produced to $H_{s o}^{h p}(\widehat{p}, \widehat{x})$ corresponding to $j=l+1 / 2$ and $j=l-1 / 2$ in the first order in two parameters $\Theta$ and $\bar{\theta}$ for hydrogenic atoms for $(n, l)$ states by applying standard perturbation theory and through the structure constants which specify the dimensionality of the GHP model of hydrogenic atoms such as $\mathrm{He}^{+}, \mathrm{Li}^{+2}$, and $\mathrm{Be}^{+}$. Thus, we obtain the following results:

$\frac{E_{s o}^{u h p}}{\epsilon N_{n l}^{2} k_{+}}=$

$=\int_{0}^{+\infty} s^{-2 \delta \eta_{n}}(1-s)^{2 l+2}\left[P_{n}^{\left(2 \eta_{n}, 2 l+1\right)}(1-2 s)\right]^{2} \times$

$\times\left[\left(\frac{b \delta^{3} s^{5 / 2}}{(1-s)^{3}}+\frac{\delta^{3} b s^{2}}{(1-s)^{2}}-\frac{a \delta^{3} s^{3 / 2}}{(1-s)^{3}}\right) \Theta+\frac{\bar{\theta}}{2 \mu}\right] d r$

$\frac{E_{s o}^{d h p}}{\epsilon N_{n l}^{2} k_{-}}=$

$=\int_{0}^{+\infty} s^{-2 \delta \eta_{n}}(1-s)^{2 l+2}\left[P_{n}^{\left(2 \eta_{n}, 2 l+1\right)}(1-2 s)\right]^{2} \times$

$\times\left[\left(\frac{b \delta^{3} s^{5 / 2}}{(1-s)^{3}}+\frac{\delta^{3} b s^{2}}{(1-s)^{2}}-\frac{a \delta^{3} s^{3 / 2}}{(1-s)^{3}}\right) \Theta+\frac{\bar{\theta}}{2 \mu}\right] d r$

We have $s=\exp (-\delta r)$, this allows us to obtain $d r=-\frac{1}{\delta} \frac{d s}{s}$. After introducing a new variable $z=$ $=1-2 s$, we have $d r=-\frac{1}{\delta} \frac{d z}{1-z}, s=\frac{1-z}{2}$ and $1-s=$ $=\frac{1+z}{2}$, the approximations (29) in that case have the following form:

$$
\begin{aligned}
& \frac{\exp (-\delta r)}{r^{2}} \approx \frac{\delta^{2} s^{2}}{(1-s)^{2}}=\frac{\delta^{2}(1-z)^{2}}{(1+z)^{2}} \\
& \frac{\exp (-\delta r)}{r^{3}} \approx \frac{\delta^{3} s^{5 / 2}}{(1-s)^{3}}=\sqrt{2} \frac{\delta^{3}(1-z)^{5 / 2}}{(1+z)^{3}} \\
& \frac{1}{r^{3}} \approx \frac{\delta^{3} s^{3 / 2}}{(1-s)^{3}}=2^{3 / 2} \frac{\delta^{3}(1-z)^{3 / 2}}{(1+z)^{3}}
\end{aligned}
$$

This allows us to reformulate Eq. (30) as follows:

$$
\begin{aligned}
& \frac{2^{2 l+2-2 \delta \eta_{n}} E_{s o}^{u h p}}{\epsilon N_{n l}^{2} k_{+}}=\int_{-1}^{+1}(1-z)^{-2 \delta \eta_{n}}(1+z)^{2 l+2} \times \\
& \times\left[P_{n}^{\left(2 \eta_{n}, 2 l+1\right)}(z)\right]^{2}\left[\left(\sqrt{2} \frac{\delta^{2} b(1-z)^{3 / 2}}{2(1+z)^{3}}+\right.\right. \\
& \left.\left.+\frac{\delta^{2} b(1-z)}{2(1+z)^{2}}-\frac{a \delta^{3} s^{3 / 2}}{(1-s)^{3}}\right) \Theta+\frac{\bar{\theta}}{2 \mu \delta} \frac{1}{1-z}\right] d z
\end{aligned}
$$

ISSN 2071-0194. Ukr. J. Phys. 2020. Vol. 65, No. 11 
$\frac{2^{2 l+2-2 \delta \eta_{n}} E_{s o}^{d h p}}{\epsilon N_{n l}^{2} k_{+}}=\int_{-1}^{+1}(1-z)^{-2 \delta \eta_{n}}(1+z)^{2 l+2} \times$

$\times\left[P_{n}^{\left(2 \eta_{n}, 2 l+1\right)}(z)\right]^{2}\left[\left(\sqrt{2} \frac{\delta^{2} b(1-z)^{3 / 2}}{2(1+z)^{3}}+\right.\right.$

$\left.\left.+\frac{\delta^{2} b(1-z)}{2(1+z)^{2}}-\frac{a \delta^{3} s^{3 / 2}}{(1-s)^{3}}\right) \Theta+\frac{\bar{\theta}}{2 \mu \delta} \frac{1}{1-z}\right] d z$.

A direct simplification gives nonrelativistic energy corrections $E_{s o}^{u h p}$ and $E_{s o}^{d h p}$ in the first order in two infinitesimal parameters $\Theta$ and $\bar{\theta}$ for the hydrogenic atoms such as $\mathrm{He}^{+}, \mathrm{Li}^{+2}$, and $\mathrm{Be}^{+}$as follows:

$$
\begin{aligned}
& E_{\text {so }}^{u h p}\left(k_{+}, n, a, b, \delta, j, l, s\right)= \\
& =\epsilon N_{n l}^{2} k_{+}\left[\Theta \sum_{i=1}^{3} T_{i}(n, l, \delta)+\frac{\bar{\theta}}{2 \mu} T_{4}(n, l, \delta)\right], \\
& E_{s o}^{d h p}\left(k_{+}, n, a, b, \delta, j, l, s\right)= \\
& =\epsilon N_{n l}^{2} k_{-}\left[\Theta \sum_{i=1}^{3} T_{i}(n, l, \delta)+\frac{\bar{\theta}}{2 \mu} T_{4}(n, l, \delta)\right],
\end{aligned}
$$

where the factors $T_{i}(n, l, \delta)$ are given by:

$$
\begin{aligned}
& \frac{2^{2 l+3-2 \delta \eta_{n}} T_{1}(n, l, \delta)}{\sqrt{2} \delta^{2} b}=\int_{-1}^{+1}(1-z)^{-2 \delta \eta_{n}+3 / 2}(1+z)^{2 l-1} \times \\
& \times\left[P_{n}^{\left(2 \eta_{n}, 2 l+1\right)}(z)\right]^{2} d z \\
& \frac{2^{2 l+3-2 \delta \eta_{n}} T_{2}(n, l, \delta)}{\delta^{2} b}=\int_{-1}^{+1}(1-z)^{-2 \delta \eta_{n}+1}(1+z)^{2 l} \times \\
& \times\left[P_{n}^{\left(2 \eta_{n}, 2 l+1\right)}(z)\right]^{2} d z, \\
& \frac{2^{2 l+3-2 \delta \eta_{n}} T_{3}(n, l, \delta)}{2^{3 / 2} \delta^{2} a}=\int_{-1}^{+1}(1-z)^{-2 \delta \eta_{n}+1 / 2}(1+z)^{2 l-1} \times \\
& \times\left[P_{n}^{\left(2 \eta_{n}, 2 l+1\right)}(z)\right]^{2} d z, \\
& \delta 2^{2 l+2-2 \delta \eta_{n}} T_{4}(n, l, \delta)=\int_{-1}^{+1}(1-z)^{-2 \delta \eta_{n}-1}(1+z)^{2 l+2} \times \\
& \times\left[P_{n}^{\left(2 \eta_{n}, 2 l+1\right)}(z)\right]^{2} d z .
\end{aligned}
$$

For the ground state $n=0$, we have $P_{0}^{\left(2 \eta_{n}, 2 l+1\right)}(z)=1$. Thus, the above four factors in ISSN 2071-0194. Ukr. J. Phys. 2020. Vol. 65, No. 11
Eq. (34) are reduced to the following simple form:

$$
\begin{aligned}
& T_{1}(n, l, \delta)=\frac{\sqrt{2} \delta^{2} b}{2^{2 l+3-2 \delta \eta_{0}}} \int_{-1}^{+1}(1-z)^{-2 \delta \eta_{0}+3 / 2}(1+z)^{2 l-1} d z \\
& T_{2}(0, l, \delta)=\frac{\delta^{2} b}{2^{2 l+3-2 \delta \eta_{0}}} \int_{-1}^{+1}(1-z)^{-2 \delta \eta_{0}+1}(1+z)^{2 l} d z \\
& T_{3}(0, l, \delta)=\frac{2^{3 / 2} \delta^{2} a}{2^{2 l+3-2 \delta \eta_{0}}} \int_{-1}^{+1}(1-z)^{-2 \delta \eta_{0}+1 / 2}(1+z)^{2 l-1} d z \\
& T_{4}(0, l, \delta)=\frac{1}{\delta 2^{2 l+2-2 \delta \eta_{0}}} \int_{-1}^{+1}(1-z)^{-2 \delta \eta_{0}-1}(1+z)^{2 l+2} d z
\end{aligned}
$$

Let us compare Eq. (35) with the integral of the form [46]:

$$
\begin{aligned}
& \int_{-1}^{+1}(1-z)^{\alpha}(1+z)^{\beta} P_{m}^{\alpha}(z) P_{n}^{\beta}(z) d z= \\
& =\frac{2^{\alpha+\beta+1} \Gamma(n+\alpha+1) \Gamma(n+\beta+1)}{(2 n+\alpha+\beta+1) \Gamma(2 n+\alpha+\beta+1)} \delta_{m n} \Rightarrow \\
& \Rightarrow \int_{-1}^{+1}(1-z)^{n+\alpha}(1+z)^{n+\beta} d z= \\
& =\frac{2^{2 n+\alpha+\beta+1} \Gamma(n+\alpha+1) \Gamma(n+\beta+1)}{(2 n+\alpha+\beta+1) \Gamma(2 n+\alpha+\beta+1)} .
\end{aligned}
$$

We obtain:

$$
\begin{aligned}
& \frac{2 T_{1}(0, l, \delta)}{\delta^{2} b}= \\
& =\frac{\Gamma\left(-2 \delta \eta_{0}+5 / 2\right) \Gamma(2 l)}{\left(-2 \delta \eta_{0}+2 l+3 / 2\right) \Gamma\left(-2 \delta \eta_{0}+2 l+3 / 2\right)}, \\
& \frac{2 T_{2}(0, l, \delta)}{\delta^{2} b}= \\
& =\frac{\Gamma\left(-2 \delta \eta_{0}+2\right) \Gamma(2 l+1)}{\left(-2 \delta \eta_{0}+2 l+2\right) \Gamma\left(-2 \delta \eta_{0}+2 l+2\right)}, \\
& \frac{2 T_{3}(0, l, \delta)}{\delta^{2} a}= \\
& =\frac{\Gamma\left(-2 \delta \eta_{0}+3 / 2\right) \Gamma(2 l)}{\left(-2 \delta \eta_{0}+2 l+1 / 2\right) \Gamma\left(-2 \delta \eta_{0}+2 l+1 / 2\right)}, \\
& 2 \delta T_{4}(0, l, \delta)= \\
& =\frac{\Gamma\left(-2 \delta \eta_{0}\right) \Gamma(2 l)}{\left(-2 \delta \eta_{0}+2 l+2\right) \Gamma\left(-2 \delta \eta_{0}+2 l+2\right)}
\end{aligned}
$$

with $\eta_{0}=\sqrt{\frac{\epsilon_{0}-A}{\delta^{2}}+l(l+1)}$ and $\epsilon_{0}=-2 \mu E_{0 l}$. Substituting Eqs. (37) into Eq. (33), we obtain nonrelativistic energy corrections $E_{s o}^{u h p}\left(k_{+}, 0, a, b, \delta, j, l, s\right)$

993 
and $E_{s o}^{d h p}\left(k_{-}, 0, a, b, \delta, j, l, s\right)$ for the ground state in the first order in two infinitesimal parameters $\Theta$ and $\bar{\theta}$ for the hydrogenic atoms such as $\mathrm{He}^{+}, \mathrm{Li}^{+2}$, and $\mathrm{Be}^{+}$corresponding to $j=l+1 / 2$ and $j=l-1 / 2$ as:

$$
\begin{aligned}
& E_{s o}^{u h p}\left(k_{+}, 0, a, b, \delta, j, l, s\right)= \\
& =\epsilon N_{0 l}^{2} k_{+}\left[\Theta T(0, l, \delta)+\frac{\bar{\theta}}{2 \mu} T_{4}(0, l, \delta)\right] \\
& E_{s o}^{d h p}\left(k_{+}, 0, a, b, \delta, j, l, s\right)= \\
& =\epsilon N_{0 l}^{2} k_{-}\left[\Theta T(0, l, \delta)+\frac{\bar{\theta}}{2 \mu} T_{4}(0, l, \delta)\right]
\end{aligned}
$$

with $\sum_{i=1}^{3} T_{i}(0, l, \delta)=T(n=0, l, \delta)$ and $N_{0 l}^{2}=$ $=\frac{\delta a \Gamma(a+2 l+2) l}{\Gamma(a+1) \Gamma(2 l+2)}$. For the first excited state $n=1$, we have $P_{0}^{(\alpha, \beta)}(z)=\alpha+1+(\alpha+\beta+2) \frac{z-1}{2}$, thus the four factors in Eq. (34) are reduced to the following simple form:

$$
\begin{aligned}
& T_{1}(1, l, \delta)=\frac{\sqrt{2} \delta^{2} b}{2^{2 l+3-2 \delta \eta_{1}}} \int_{-1}^{+1}(1-z)^{-2 \delta \eta_{1}+3 / 2} \times \\
& \times(1+z)^{2 l-1}\left[g_{-} h(1-z)\right]^{2} d z, \\
& T_{2}(1, l, \delta)=\frac{\delta^{2} b}{2^{2 l+3-2 \delta \eta_{1}}} \int_{-1}^{+1}(1-z)^{-2 \delta \eta_{1}+1} \times \\
& \times(1+z)^{2 l}\left[g \_h(1-z)\right]^{2} d z, \\
& T_{3}(1, l, \delta)=\frac{2^{3 / 2} \delta^{2} a}{2^{2 l+3-2 \delta \eta_{1}}} \int_{-1}^{+1}(1-z)^{-2 \delta \eta_{1}+1 / 2} \times \\
& \times(1+z)^{2 l-1}\left[g_{-} h(1-z)\right]^{2} d z, \\
& T_{4}(1, l, \delta)=\frac{1}{\delta 2^{2 l+2-2 \delta \eta_{1}}} \int_{-1}^{+1}(1-z)^{-2 \delta \eta_{1}-1} \times \\
& \times(1+z)^{2 l+2}\left[g_{-} h(1-z)\right]^{2} d z
\end{aligned}
$$

with $g=2 \delta \eta_{1}+1, \quad h=\eta_{1}+l+2, \quad \eta_{1}=$ $=\sqrt{\frac{\epsilon_{1}-A}{\delta^{2}}+l(l+1)}$ and $\epsilon_{1}=-2 \mu E_{1 l}$. A direct simplification to Eq. (39) gives:

$$
\begin{aligned}
& \frac{2^{2 l+3-2 \delta \eta_{1}} T_{1}(1, l, \delta)}{\sqrt{2} \delta^{2} b}= \\
& =g^{2} \int_{-1}^{+1}(1-z)^{-2 \delta \eta_{1}+3 / 2}(1+z)^{2 l-1} d z- \\
& -2 g h \int_{-1}^{+1}(1-z)^{-2 \delta \eta_{1}+5 / 2}(1+z)^{2 l-1} d z+
\end{aligned}
$$

994 $+h^{2} \int_{-1}^{+1}(1-z)^{-2 \delta \eta_{1}+7 / 2}(1+z)^{2 l-1} d z$, $\frac{2^{2 l+3-2 \delta \eta_{1}} T_{2}(1, l, \delta)}{\delta^{2} b}=$ $=g^{2} \int_{-1}^{+1}(1-z)^{-2 \delta \eta_{1}+1}(1+z)^{2 l} d z-$ $-2 g h \int_{-1}^{+1}(1-z)^{-2 \delta \eta_{1}+2}(1+z)^{2 l} d z+$ $+h^{2} \int_{-1}^{+1}(1-z)^{-2 \delta \eta_{1}+3}(1+z)^{2 l-1} d z$, $\frac{2^{2 l+3-2 \delta \eta_{1}} T_{3}(1, l, \delta)}{\sqrt{2} \delta^{2} b}=$ $=g^{2} \int_{-1}^{+1}(1-z)^{-2 \delta \eta_{1}+1 / 2}(1+z)^{2 l-1} d z-$ $-2 g h \int_{-1}^{+1}(1-z)^{-2 \delta \eta_{1}+3 / 2}(1+z)^{2 l-1} d z+$ $\left.+h^{2} \int_{-1}^{+1}(1-z)^{-2 \delta \eta_{1}+5 / 2}(1+z)^{2 l-1} d z\right)$, $\frac{2^{2 l+3-2 \delta \eta_{1}} T_{4}(1, l, \delta)}{\sqrt{2} \delta^{2} b}=$ $=g^{2} \int_{-1}^{+1}(1-z)^{-2 \delta \eta_{1}-1}(1+z)^{2 l+2} d z-$ $-2 g h \int_{-1}^{+1}(1-z)^{-2 \delta \eta_{1}}(1+z)^{2 l+2} d z+$ $+h^{2} \int_{-1}^{+1}(1-z)^{-2 \delta \eta_{1}+1}(1+z)^{2 l+2} d z$.

Comparing Eq. (38) with integral (36), we have four factors as:

$$
\begin{aligned}
& T_{1}(1, l, \delta)=\frac{g^{2} \delta^{2} b \Gamma\left(-2 \delta \eta_{1}+5 / 2\right) \Gamma(2 l)}{2(\gamma+3 / 2) \Gamma(\gamma+3 / 2)}- \\
& -\frac{2 g h \delta^{2} b \Gamma\left(-2 \delta \eta_{1}+7 / 2\right) \Gamma(2 l)}{2(\gamma+5 / 2) \Gamma(\gamma+5 / 2)}+ \\
& +\frac{h^{2} \delta^{2} b \Gamma\left(-2 \delta \eta_{1}+9 / 2\right) \Gamma(2 l)}{2(\gamma+7 / 2) \Gamma(\gamma+7 / 2)}
\end{aligned}
$$

ISSN 2071-0194. Ukr. J. Phys. 2020. Vol. 65, No. 11 


$$
\begin{aligned}
& T_{2}(1, l, \delta)=\frac{g^{2} \delta^{2} b \Gamma\left(-2 \delta \eta_{1}+2\right) \Gamma(2 l+1)}{2(\gamma+2) \Gamma(\gamma+2)}- \\
& -\frac{2 g h \delta^{2} b \Gamma\left(-2 \delta \eta_{1}+3\right) \Gamma(2 l+1)}{(\gamma+3) \Gamma(\gamma+3)}+ \\
& +\frac{h^{2} \delta^{2} b \Gamma\left(-2 \delta \eta_{1}+4\right) \Gamma(2 l+1)}{(\gamma+4) \Gamma(\gamma+4)}, \\
& T_{3}(1, l, \delta)=\frac{-g^{2} \delta^{2} a \Gamma\left(-2 \delta \eta_{1}+3 / 2\right) \Gamma(2 l)}{2(\gamma+1 / 2) \Gamma(\gamma+1 / 2)}+ \\
& +\frac{2 g h \delta^{2} a \Gamma\left(-2 \delta \eta_{1}+5 / 2\right) \Gamma(2 l)}{(\gamma+3 / 2) \Gamma(\gamma+3 / 2)}- \\
& -\frac{4 h^{2} \delta^{2} a \Gamma\left(-2 \delta \eta_{1}+7 / 2\right) \Gamma(2 l)}{(\gamma+5 / 2) \Gamma(\gamma+5 / 2)} \\
& T_{4}(1, l, \delta)=\frac{g^{2} \Gamma\left(-2 \delta \eta_{1}\right) \Gamma(2 l+3)}{\delta(\gamma+2) \Gamma(\gamma+2)}- \\
& -\frac{4 g h \Gamma\left(-2 \delta \eta_{1}+1\right) \Gamma(2 l+2)}{\delta(\gamma+3) \Gamma(\gamma+3)}+ \\
& +\frac{4 h^{2} b \Gamma\left(-2 \delta \eta_{1}+2\right) \Gamma(2 l)}{\delta(\gamma+3) \Gamma(\gamma+3)}
\end{aligned}
$$

with $\gamma=-2 \delta \eta_{1}+2$. This allows us to obtain nonrelativistic energy corrections for the first excited state $E_{s o}^{u h p}\left(k_{+}, 1, a, b, \delta, j, l, s\right)$ and $E_{s o}^{d h p}\left(k_{-}, 1, a, b, \delta, j, l, s\right)$ for the first excited state in the first order in two infinitesimal parameters $\Theta$ and $\bar{\theta}$ for the hydrogenic atoms such as $\mathrm{He}^{+}, \mathrm{Li}^{+2}$ and $\mathrm{Be}^{+}$corresponding to $j=l+1 / 2$ and $j=l-1 / 2$ as:

$$
\begin{aligned}
& E_{s o}^{u h p}\left(k_{+}, 1, a, b, \delta, j, l, s\right)= \\
& =\epsilon N_{1 l}^{2} k_{+}\left[\Theta T(1, l, \delta)+\frac{\bar{\theta}}{2 \mu} T_{4}(1, l, \delta)\right], \\
& E_{s o}^{d h p}\left(k_{+}, 1, a, b, \delta, j, l, s\right)= \\
& =\epsilon N_{1 l}^{2} k_{-}\left[\Theta T(1, l, \delta)+\frac{\bar{\theta}}{2 \mu} T_{4}(1, l, \delta)\right]
\end{aligned}
$$

with $\sum_{i=1}^{3} T_{i}(n, l, \delta)=T(n=1, l, \delta)$ and $N_{1 l}^{2}=$ $\frac{\delta a \Gamma(a+2 l+3) l}{\Gamma(a+2) \Gamma(2 l+3)}$. Thus, for any excited state, the nonrelativistic energy corrections $E_{s o}^{u h p}\left(k_{+}, n, a, b, \delta, j, l, s\right)$ and $E_{s o}^{d h p}\left(k_{-}, n, a, b, \delta, j, l, s\right)$ for the $n^{t h}$ excited state in the first order in two infinitesimal parameters $\Theta$ and $\bar{\theta}$ for the hydrogenic atoms such as $\mathrm{He}^{+}, \mathrm{Li}^{+2}$, and $\mathrm{Be}^{+}$corresponding to $j=l+1 / 2$ and $j=l-1 / 2$ as:

$$
\begin{aligned}
& E_{s o}^{u h p}\left(k_{+}, n, a, b, \delta, j, l, s\right)= \\
& =\epsilon N_{n l}^{2} k_{+}\left[\Theta T(n, l, \delta)+\frac{\bar{\theta}}{2 \mu} T_{4}(n, l, \delta)\right],
\end{aligned}
$$

ISSN 2071-0194. Ukr. J. Phys. 2020. Vol. 65, No. 11
$E_{s o}^{d h p}\left(k_{+}, n, a, b, \delta, j, l, s\right)=$

$=\epsilon N_{n l}^{2} k_{-}\left[\Theta T(n, l, \delta)+\frac{\bar{\theta}}{2 \mu} T_{4}(n, l, \delta)\right]$

with $\sum_{i=1}^{3} T_{i}(n, l, \delta)=T(n, l, \delta)$. This allow us to obtain the following important physical results for hydrogenic atoms such as $\mathrm{He}^{+}, \mathrm{Li}^{+2}$, and $\mathrm{Be}^{+}$in the GHP model:

$$
\begin{aligned}
& H_{s o}^{h p}(\widehat{p}, \widehat{x}) \frac{R_{n l}(r)}{r} Y_{l}^{m}(\theta, \varphi)= \\
& =\left\{\begin{array}{l}
E_{s o}^{u h p}\left(k_{+}, n, a, b, \delta, j, l, s\right) \frac{R_{n l}(r)}{r} Y_{l}^{m}(\theta, \varphi) \\
\text { for } j=l+1 / 2, \\
E_{s o}^{d h p}\left(k_{+}, n, a, b, \delta, j, l, s\right) \frac{R_{n l}(r)}{r} Y_{l}^{m}(\theta, \varphi) \\
\text { for } j=l+1 / 2 .
\end{array}\right.
\end{aligned}
$$

\subsection{Bound-state solution \\ for the modified Zeeman effect in the GHP model}

In this subsection, we will obtain the energy spectrum $E_{s o}^{u h p}\left(k_{+}, n, a, b, \delta, j, l, s\right)$ and $E_{s o}^{d \mathrm{hp}}\left(k_{-}, n, a, b, \delta, j, l, s\right)$ which is produced to $H_{s o}^{h p}(\widehat{p}, \widehat{x})$ corresponding to $j=l+1 / 2$ and $j=l-1 / 2$ in the first order in two parameters $\Theta$ and $\bar{\theta}$ for hydrogenic atoms for $(n, l)$ states. As is known, it is possible to obtain the additional symmetry for the GHP model. This physical phenomenon is induced automatically from the influence of an external uniform magnetic field $\mathbf{B}$, if we make the following two simultaneous transformations to ensure that the previous calculations are not reputed:

$(\Theta, \bar{\theta}) \rightarrow(\lambda, \bar{\sigma}) B$

Here, $\lambda$ and $\bar{\sigma}$ are just two infinitesimal real proportional constants. To simplify calculations without compromising the physical content, we choose the magnetic field $\mathbf{B}=B \mathbf{k}$. Then we transform the spinorbit coupling to the new physical phenomena as follows:

$$
\begin{aligned}
& {\left[\left(\frac{b \exp (-\delta r)}{2 r^{3}}+\frac{\delta b \exp (-\delta r)}{2 r^{2}}-\frac{a}{2 r^{3}}\right) \boldsymbol{\Theta}+\frac{\overline{\boldsymbol{\theta}}}{2 \mu}\right] \mathbf{L} \Rightarrow} \\
& \Rightarrow\left[\left(\frac{b \exp (-\delta r)}{2 r^{3}}+\frac{\delta b \exp (-\delta r)}{2 r^{2}}-\frac{a}{2 r^{3}}\right) \lambda+\frac{\bar{\sigma}}{2 \mu}\right] B L_{z} .
\end{aligned}
$$


This allowed us to derive the modified magnetic Hamiltonian operator $H_{z}^{h p}(r, \lambda, \bar{\sigma})$ for hydrogenic atoms in the GHP model in global (NC: 3D-RSP) symmetries as:

$$
\begin{aligned}
& H_{z}^{h p}(r, \lambda, \bar{\sigma})=\left[\left(\frac{b \exp (-\delta r)}{2 r^{3}}+\frac{\delta b \exp (-\delta r)}{2 r^{2}}-\right.\right. \\
& \left.\left.-\frac{a}{2 r^{3}}\right) \lambda+\frac{\bar{\sigma}}{2 \mu}\right] H_{\bmod -z .}
\end{aligned}
$$

Here, $H_{\bmod -z}=\mathbf{B J}_{-} H_{z}$ denotes the to Zeeman effect in NC quantum mechanics, while $H_{z}=$ $=-\mathbf{B S}$ is the ordinary Zeeman effect. To obtain the exact NC magnetic modifications of the energy for the ground state, the first excited state and $n^{t h}$ excited states of hydrogenic atoms such as $\mathrm{He}^{+}, \mathrm{Li}^{+2}$, and $\mathrm{Be}^{+}$in the GHP model $E_{\text {mag }}^{\mathrm{hp}}(0, a, b, \delta, j, m, s)$, $E_{\text {mag }}^{h p}(1, a, b, \delta, j, m, s)$ and $E_{\text {mag }}^{h p}(n, a, b, \delta, j, m, s)$ we just replace $k_{+}$or $k_{-}$and $(\Theta, \bar{\theta})$ in Eqs. (38), (42) and (43) by the following parameters $m$ and $(\lambda, \bar{\sigma}) B$, respectively:

$$
\begin{aligned}
& E_{\mathrm{mag}}^{h p}(0, a, b, \delta, j, m, s)= \\
& =\epsilon N_{0 l}^{2} B\left[\lambda T(0, l, \delta)+\frac{\bar{\sigma}}{2 \mu} T_{4}(0, l, \delta)\right] m, \\
& E_{\mathrm{mag}}^{h p}(1, a, b, \delta, j, m, s)= \\
& =\epsilon N_{1 l}^{2} B\left[\lambda T(1, l, \delta)+\frac{\bar{\sigma}}{2 \mu} T_{4}(1, l, \delta)\right] m, \\
& E_{\mathrm{mag}}^{h p}(n, a, b, \delta, j, m, s)= \\
& =\epsilon N_{n l}^{2} B\left[\lambda T(n, l, \delta)+\frac{\bar{\sigma}}{2 \mu} T_{4}(n, l, \delta)\right] m .
\end{aligned}
$$

We have $(-l \leq m \leq+l)$, which allow us to fix $(2 l+1)$ values for the discrete number $m$. It should be noting that the results obtained in Eq. (48) can be found by the direct calculation $E_{\text {mag }}^{h p}(n, a, b, \delta, j, m, s)=$ $=\left\langle\Psi\left|H_{z}^{h p}(r, \lambda, \bar{\sigma})\right| \Psi\right\rangle$ that takes the following explicit relation:

$$
\begin{aligned}
& E_{\text {mag }}^{h p}=\epsilon N_{n l}^{2} B m \int_{0}^{+\infty} s^{-2 \delta \eta_{n}}(1-s)^{2 l+2} \times \\
& \times\left[P_{n}^{\left(2 \eta_{n}, 2 l+1\right)}(1-2 s)\right]^{2} \times \\
& \times\left[\left(\frac{b \delta^{3} s^{5 / 2}}{(1-s)^{3}}+\frac{\delta^{3} b s^{2}}{(1-s)^{2}}-\frac{a \delta^{3} s^{3 / 2}}{(1-s)^{3}}\right) \lambda+\frac{\bar{\sigma}}{2 \mu}\right] d r .
\end{aligned}
$$

Moreover, we apply the same steps that we saw in the previous subsection to get the results presented in Eq. (48). We end this subsection by addressing the important results:

$$
\begin{aligned}
& H_{z}^{h p}(r, \lambda, \bar{\sigma}) \frac{R_{n l}(r)}{r} Y_{l}^{m}(\theta, \varphi)= \\
& =E_{\mathrm{mag}}^{h p}(n, a, b, \delta, j, m, s) \frac{R_{n l}(r)}{r} Y_{l}^{m}(\theta, \varphi) .
\end{aligned}
$$

\section{Results and Discussion}

In the previous subsections, we obtained the solution of the modified Schrödinger equation for the GHP model, which is described by the Hamiltonian operator given in Eq. (19) by using the generalized Bopp's shift method and standard perturbation theory. The energy eigenvalues are calculated in the 3D space-phase. The modified eigenenergies for the ground state, the first excited state. and $n^{\text {th }}$ excited states of hydrogenic atoms such as $\mathrm{He}^{+}, \mathrm{Li}^{+2}$, and $\mathrm{Be}^{+}$in the GHP model $E_{n c}^{u \mathrm{hp}}(0, a, b, \delta, j, l, m, s)$, $E_{n c}^{u \mathrm{hp}}(1, a, b, \delta, j, l, m, s)$ and $E_{n c}^{u \mathrm{hp}}(n, a, b, \delta, j, l, m, s)$ with spin- $1 / 2$ are obtained in this paper on the basis of our original results presented in Eqs. (38), (42), (43) and (42), in addition to the ordinary energy for the Hellmann potential model which is presented in Eq. (9) in the form:

$$
\begin{aligned}
& E_{n c}^{h p}(0, a, b, \delta, j, l, m, s)= \\
& =E_{0 l+}+\epsilon N_{0 l}^{2} B\left[\lambda T(0, l, \delta)+\frac{\bar{\sigma}}{2 \mu} T_{4}(0, l, \delta)\right] m+ \\
& +\epsilon N_{0 l}^{2}\left\{\begin{array}{l}
k_{+}\left[\Theta T(0, l, \delta)+\frac{\bar{\theta}}{2 \mu} T_{4}(0, l, \delta)\right] \\
\text { for } j=l+1 / 2, \\
k_{-}\left[\Theta T(0, l, \delta)+\frac{\bar{\theta}}{2 \mu} T_{4}(0, l, \delta)\right] \\
\text { for } j=l-1 / 2,
\end{array}\right. \\
& E_{n c}^{h p}(1, a, b, \delta, j, l, m, s)= \\
& =E_{1 l+} \epsilon N_{1 l}^{2} B\left[\lambda T(1, l, \delta)+\frac{\bar{\sigma}}{2 \mu} T_{4}(1, l, \delta)\right] m+ \\
& +\epsilon N_{1 l}^{2}\left\{\begin{array}{l}
k_{+}\left[\Theta T(1, l, \delta)+\frac{\bar{\theta}}{2 \mu} T_{4}(1, l, \delta)\right] \\
\text { for } j=l+1 / 2, \\
k_{-}\left[\Theta T(1, l, \delta)+\frac{\bar{\theta}}{2 \mu} T_{4}(1, l, \delta)\right] \\
\text { for } j=l-1 / 2
\end{array}\right.
\end{aligned}
$$

and

$E_{n c}^{h p}(n, a, b, \delta, j, l, m, s)=$

ISSN 2071-0194. Ukr. J. Phys. 2020. Vol. 65, No. 11 
$=-\frac{\delta^{2}}{2 \mu}\left[\left(\frac{(2 \mu / a)(a-b)-(n+l+1)^{2}-l(l+1)}{2(n+l+1)}\right)^{2}+\right.$ $\left.+l(l+1)+\frac{2 \mu a}{\delta}\right]+$

$+\epsilon N_{1 l}^{2} B\left[\lambda T(n, l, \delta)+\frac{\bar{\sigma}}{2 \mu} T_{4}(n, l, \delta)\right] m+$

$+\epsilon N_{1 l}^{2}\left\{\begin{array}{l}k_{+}\left[\Theta T(n, l, \delta)+\frac{\bar{\theta}}{2 \mu} T_{4}(n, l, \delta)\right] \\ \text { for } j=l+1 / 2, \\ k_{-}\left[\Theta T(n, l, \delta)+\frac{\bar{\theta}}{2 \mu} T_{4}(n, l, \delta)\right] \\ \text { for } j=l-1 / 2 .\end{array}\right.$

where the energies of the ground state $E_{0 l}$ and the first excited state $E_{1 l}$ in the symmetries of quantum mechanics in the Hellmann potential model are as follows:

$$
\begin{aligned}
& E_{0 l}=-\frac{\delta^{2}}{2 \mu}\left[\left(\frac{(2 \mu / a)(a-b)-(l+1)^{2}-l(l+1)}{2(l+1)}\right)^{2}+\right. \\
& \left.+l(l+1)+\frac{2 \mu a}{\delta}\right], \\
& E_{1 l}=-\frac{\delta^{2}}{2 \mu}\left[\left(\frac{(2 \mu / a)(a-b)-(l+2)^{2}-l(l+1)}{2(l+2)}\right)^{2}+\right. \\
& \left.+l(l+1)+\frac{2 \mu a}{\delta}\right] .
\end{aligned}
$$

Thus, the total energy $E_{n c}^{h p}(n, a, b, \delta, j, l, m, s)$ for the GHP model in (NC: 3D-RSP) symmetries is the sum of the ordinary part of the energy $E_{\mathrm{mag}}^{h p}(n, a, b, \delta, j, m, s)$ and the two corrections $E_{s o}^{u h p}\left(k_{+}, n, a, b, \delta, j, l, s\right)$ and $E_{s o}^{d h p}\left(k_{-}, n, a, b, \delta, j, l, s\right)$ (for $j=l+1 / 2$ and $j=l-1 / 2$ ) and. This is one of the main objectives of our research. Finally, we end this section by introducing the important result of this work as:

$$
\begin{aligned}
& \left(H_{h p}(p, x)+H_{s o}^{h p}(\widehat{p}, \widehat{x})+H_{z}^{h p}(r, \lambda, \bar{\sigma})\right) \times \\
& \times \frac{R_{n l}(r)}{r} Y_{l}^{m}(\theta, \varphi)= \\
& =\left(E_{n l}+\epsilon N_{n l}^{2} B\left[\lambda T(n, l, \delta)+\frac{\bar{\sigma}}{2 \mu} T_{4}(n, l, \delta)\right] m+\right. \\
& +\left\{\begin{array}{l}
E_{\text {so }}^{u h p}\left(k_{+}, n, a, b, \delta, j, l, s\right) \\
\text { for } j=l+1 / 2, \\
E_{\text {so }}^{d h p}\left(k_{+}, n, a, b, \delta, j, l, s\right) \\
\text { for } j=l+1 / 2
\end{array} \quad \frac{R_{n l}(r)}{r} Y_{l}^{m}(\theta, \varphi)\right) \cdot(53
\end{aligned}
$$

This is one of the main motivations for the topic of this work. It is clear that the obtained eigenvalues of energies are real, which allow us to consider the NC diagonal Hamiltonian $H_{n c}^{h p}(r, \Theta, \bar{\theta} \lambda, \bar{\sigma})$ as a Hermitian operator. In addition with regard for results (20), (22), and (47), the global Hamiltonian operator in the first order in and within the GHP model for hydrogenic atoms for $(n, l)$ states takes the form:

$$
\begin{aligned}
& H_{n c}^{h p}(r, \Theta, \bar{\theta} \lambda, \bar{\sigma})=-\frac{\Delta}{2 \mu}-\frac{a}{r}+\frac{b \exp (-\delta r)}{r}+ \\
& +\left[\left(\frac{b \exp (-\delta r)}{2 r^{3}}+\frac{\delta b \exp (-\delta r)}{2 r^{2}}-\frac{a}{2 r^{3}}\right) \lambda+\right. \\
& \left.+\frac{\bar{\sigma}}{2 \mu}\right] H_{\bmod -z}+ \\
& +\epsilon\left[\left(\frac{b \exp (-\delta r)}{2 r^{3}}+\frac{\delta b \exp (-\delta r)}{2 r^{2}}-\frac{a}{2 r^{3}}\right) \Theta+\frac{\bar{\theta}}{2 \mu}\right] \mathbf{L S .}
\end{aligned}
$$

This is the equation for hydrogenic atoms under the influence of the GHP model interactions. It should be pointed out that this treatment considers only the first-order terms in either $\Theta$ or $\bar{\theta}$. Clearly, the first part of Eq. (54) presents the Hamiltonian operator in the ordinary quantum mechanics for Hellmann's potential model, while the second and third parts, respectively, present the spin-orbit and modified Zeeman Hamiltonian operators for the GHP model, which are induced automatically by the NC properties of space and phase. It is evident to consider the atomic quantum number $m$ can take $(2 l+1)$ values, and we have also two values for $j=l+1 / 2$ and $j=l-1 / 2$ corresponding to up and down polarities, thus, every state in the usual 3D-space of energy for the GHP model will be $2(2 l+1)$ substate in (NC: 3D-RSP). Thus, the total complete degeneracy of the obtained energy level of the GHP model is obtained as a sum of all allowed values of $l$. The total degeneracy is, thus,

$\sum_{l=1}^{n-1} 2(2 l+1)=2 n^{2}$.

In the limit $(\Theta, \bar{\theta}) \rightarrow(0,0)$, we have

$\operatorname{Lim}_{(\Theta, \bar{\theta}) \rightarrow(0,0)} E_{n c}^{h p}(n, a, b, \delta, j, l, m, s)=E_{n l}$.

Now, we can deduce the energy levels $E_{n c}^{h p}(n, k(j, l, s), a, b, \delta, j, l, m, s)$ of neutral atoms (the 
atoms that possess a number of external electrons equal to the number of protons in the nucleus) in the GHP model such as sodium, carbon, and gold atoms, which are known by their chemical symbols (Na, C and $\mathrm{Au})$, respectively. We just replace $k_{+}(j, l, s)$ with the new value $k(j, l, s)=j(j+1)-l(l+1)-s(s+1)$ in Eq. (51.3) to ensure that previous calculations are not reputed:

$E_{n c}^{h p}(n, k(j, l, s), a, b, \delta, j, l, m, s)=$

$=-\frac{\delta^{2}}{2 \mu}\left[\left(\frac{(2 \mu / a)(a-b)-(n+l+1)^{2}-l(l+1)}{2(n+l+1)}\right)^{2}+\right.$

$\left.+l(l+1)+\frac{2 \mu a}{\delta}\right]+$

$+\epsilon N_{1 l}^{2} B\left[\lambda T(n, l, \delta)+\frac{\bar{\sigma}}{2 \mu} T_{4}(n, l, \delta)\right] m+$

$+\epsilon N_{n l}^{2} k(j, l, s)\left[\Theta T(n, l, \delta)+\frac{\bar{\theta}}{2 \mu} T_{4}(n, l, \delta)\right]$.

We now look at some special cases and relationships between our recent results and some other existing results in our previous works.

Case 1: First case where we set $a=Z e^{2}$ and $b=0$, the GHP model is reduced to the modified Coulomb potential, it is easy to show that Eqs. (19), (39), and (44) are reduced to the modified interaction $H_{\text {pert }}^{\text {col }}(r, \Theta, \bar{\theta})$ of a particle in the modified Coulomb potential and the corresponding NC spectrum, respectively:

$H_{\text {pert }}^{h p}(r, \Theta, \bar{\theta}) \rightarrow H_{\text {pert }}^{\text {col }}(r, \Theta, \bar{\theta})=$

$=-\frac{Z e^{2}}{2 r^{3}} \mathbf{L} \Theta+\frac{\mathbf{L} \overline{\boldsymbol{\theta}}}{2 \mu}$

and

$E_{n c}^{h p} \rightarrow E_{n c}^{h p}=-\frac{Z^{2} \mu e^{2}}{2(n+l+1)}+$

$+\epsilon N_{1 l}^{2} B\left[\lambda T(n, l, b=0, \delta)+\frac{\bar{\sigma}}{2 \mu} T_{4}(n, l, b=0, \delta)\right] m+$

$+\epsilon N_{1 l}^{2}\left\{\begin{array}{l}k_{+}\left[\Theta T(n, l, b=0, \delta)+\frac{\bar{\theta}}{2 \mu} T_{4}(n, l, b=0, \delta)\right] \\ \text { for } j=l+1 / 2, \\ k_{-}\left[\Theta T(n, l, b=0, \delta)+\frac{\bar{\theta}}{2 \mu} T_{4}(n, l, b=0, \delta)\right] \\ \text { for } j=l-1 / 2 .\end{array}\right.$
Case 2: Similarly, if we set $a=0$ and $b$ different from zero, Eqs. (19) and (39) are reduced to the results of modified Yukawa $H_{\text {pert }}^{\text {Yukawa }}(r, \Theta, \bar{\theta})$ and correspond to a modified bound state energy spectrum of a vibrating rotating diatomic molecule, respectively:

$$
\begin{aligned}
& H_{\text {pert }}^{\text {col }}(r, \Theta, \bar{\theta}) \rightarrow H_{\text {pert }}^{\text {Yukawa }}(r, \Theta, \bar{\theta})+\frac{\mathbf{L} \overline{\boldsymbol{\theta}}}{2 \mu}= \\
& =\left(\frac{b \exp (-\delta r)}{2 r^{3}}+\frac{\delta b \exp (-\delta r)}{2 r^{2}}\right) \mathbf{L} \Theta
\end{aligned}
$$

and

$$
\begin{aligned}
& E_{n c}^{h p} \rightarrow E_{n c}^{\text {Yukawa }}(n, b, \delta, j, l, m, s)= \\
& =-\frac{\delta^{2}}{2 \mu}\left[\left(\frac{-(2 \mu / a) b-(n+l+1)^{2}-l(l+1)}{2(n+l+1)}\right)^{2}+\right. \\
& +l(l+1)]+ \\
& +\epsilon N_{1 l}^{2} B\left[\lambda T(n, l, b=0, \delta)+\frac{\bar{\sigma}}{2 \mu} T_{4}(n, l, b=0, \delta)\right] m+ \\
& +\epsilon N_{1 l}^{2}\left\{\begin{array}{l}
k_{+}\left[\Theta T(n, l, a=0, \delta)+\frac{\bar{\theta}}{2 \mu} T_{4}(n, l, a=0, \delta)\right] \\
\text { for } j=l+1 / 2, \\
k_{-}\left[\Theta T(n, l, a=0, \delta)+\frac{\bar{\theta}}{2 \mu} T_{4}(n, l, a=0, \delta)\right] \\
\text { for } j=l-1 / 2 .
\end{array}\right.
\end{aligned}
$$

It is possible to recover the results for the commutative space, when we consider $(\Theta, \bar{\theta})$ equal $(0,0)$.

\section{Conclusion}

In this paper, the three-dimensional modified radial Schrödinger equation has been solved in the GHP model by using the improved approximation scheme to the centrifugal term for any l-states and generalized Bopp's shift method, in addition to standard perturbation theory in (NC: 3D-RSP) symmetries. We resume the main obtained results:

The energy eigenvalues of the bound states of hydrogenic atoms in the GHP model $\left(E_{n c}^{u h p}(0, a, b, \delta, j, l, m, s), \quad E_{n c}^{u h p}(1, a, b, \delta, j, l, m, s)\right.$ and $\left.E_{n c}^{u h p}(n, a, b, \delta, j, l, m, s)\right)$ with spin-1/2 for the ground state, the first excited state, and $n^{\text {th }}$ excited states have been analytically found. The energy eigenvalues depending on $(a, b, \delta)$ parameters and the discrete atomic quantum numbers $(j, l, m, s)$ have a finite number of the quantized energy spectrum for the GHP model.

ISSN 2071-0194. Ukr. J. Phys. 2020. Vol. 65, No. 11 
The energy eigenvalues of the bound states of neutral atoms such as $\mathrm{Na}, \mathrm{C}$, and $\mathrm{Au}$ in the GHP model, with spin-S for excited states have been analytically found. The energy eigenvalues $E_{n c}^{h p}(n, k(j, l, s), a, b, \delta, j, l, m, s)$ depending on $(a, b, \delta)$ parameters and the discrete atomic quantum numbers $(j, l, m, s)$ are obtained.

The ordinary kinetic term $-\frac{\Delta}{2 \mu}$ is modified to the new form $\left(-\frac{\Delta}{2 \mu} \frac{\mathbf{L} \overline{\boldsymbol{\theta}}}{2 \mu}-\frac{\mathbf{L} \overline{\boldsymbol{\sigma}}}{2 \mu}\right)$ for the GHP model in (NC: 3D-RSP) symmetries,

The Hamiltonian operator in (NC: 3D-RSP) symmetries $H_{n c}^{h p}(r, \Theta, \bar{\theta}, \lambda, \bar{\sigma})$ is the sum of the Hamiltonian operator of the HP model $H_{h p}(p, x)$ and two operators, the first one is the modified spin-orbit interaction $H_{s o}^{h p}(\widehat{p}, \widehat{x})$, while the second is a modified Zeeman operator $H_{z}^{\mathrm{hp}}(r, \lambda, \bar{\sigma})$ for the hydrogenic atoms and neutral atoms.

It has been shown that the MSE presents useful rich spectra for the improved understanding of the hydrogenic atoms such as $\mathrm{He}^{+}, \mathrm{Li}^{+2}$, and $\mathrm{Be}^{+}$and neutral atoms such as $\mathrm{Na}, \mathrm{C}$, and $\mathrm{Au}$ influenced by the GHP model. We have seen also that the modified spin-orbital interaction and the modified Zeeman effect appeared due to the presence of the two infinitesimal parameters $(\Theta, \bar{\theta})$ which are induced by (position-position) and (phase-phase) noncommutativity property of space-phase. It should be noted that the results obtained in this research would be identical with corresponding results in ordinary quantum mechanics (see, e.g., [3-8]), when the two parameters are reduced to the limited values.

This work has been partly supported by the Algerian Ministry of Higher Education and Scientific Research and DGRST under Project No. B00L02UN280120180001 and by the Laboratory of Physics and Material Chemistry of the University of M'sila. The author is grateful to the reviewers for comments, which have significantly helped for the improvement of this present research work.

1. S.M. Ikhdair, R. Server. A perturbative Ptratment for the energy levels of neutral atoms. Intern. J. Mod. Phys. A 21 (31), 6465 (2006).

2. H. Hellmann. A new approximation method in the problem of many electrons. J. Chem. Phys. 3 (1), 61 (1935).

3. H. Hellmann, W. Kassatotschkin. Metallic bonding according to the combined approximation procedure. J. Chem. Phys. 4 (5), 324 (1936).
4. G. Kocak, O. Bayrak, I. Boztosun. Arbitrary l-state solution of the Hellmann potential. J. Theor. Comput. Chem. 6 (4), 893 (2007).

5. S.M. Ikhdair, B.J. Falaye. Two approximate analytic eigensolutions of the Hellmann potential with any arbitrary angular momentum. Zeitschrift Für Naturforschung A 68(10-11) (2013).

6. M. Hamzavi, K.E. Thylwe, A.A. Rajabi. Approximate bound states solution of the Hellmann potential. Commun. Theor. Phys. 60 (1), 1 (2013).

7. C.A. Onate, M.C. Onyeaju, A.N. Ikot, O. Ebomwonyi. Eigen solutions and entropic system for Hellmann potential in the presence of the Schrödinger equation. Eur. Phys. J. Plus. 132 (11), 462 (2017).

8. C.O. Edet, K.O. Okorie, H. Louis, N.A. Nzeata-Ibe. Any lstate solutions of the Schrödinger equation interacting with Hellmann-Kratzer potential model. Indian J. Phys. 94243 (2020).

9. H. Louis, I.B. Iserom, M.T. Odey, A.U. Ozioma, N.-I. Nelson, I.I. Alexander, E.C. Okon, Solutions to the Dirac equation for Manning-Rosen plus shifted Deng-Fan potential and Coulomb-like tensor interaction using NikiforovUvarov method. Intern. J. Chem. 10 (3), 99 (2018).

10. H. Louis, I. Iserom, O.U. Akakuru, N.A. Nzeala-ibe, A.I. Ikeuba, T.O. Magu, P. Amos, E.O. Collins. $l$-state solutions of the relativistic and non- relativistic wave equations for modified Hylleraas-Hulthen potential using the Nikiforov-Uvarov quantum formalism. Oriental J. Phys. Sci. 3 (1), 3 (2018).

11. L. Hitler, I.B. Iserom, P. Tchoua, A.A. Ettah. Bound state solutions of the Klein-Gordon equation for the more general exponential screened Coulomb potential plus Yukawa (MGESCY) potential using Nikiforov-Uvarov method. J. Phys. Math. 9 (01), 1000261 (2018).

12. C.O. Edet, P.O Okoi, S.O. Chima. Analytic solutions of the Schrödinger equation with non-central generalized inverse quadratic Yukawa potential. Revista Brasileira de Ensino de Fisica. 42, e20190083 (2020).

13. C.O. Edet, P.O. Okoi. Any l-state solutions of the Schrödinger equation for q-deformed Hulthen plus generalized inverse quadratic Yukawa potential in arbitrary dimensions. Revista Mexicana de Fisica 65, 333 (2019).

14. U.S. Okorie, A.N. Ikot, C.O. Edet, I.O. Akpan, R. Sever, R. Rampho. Solutions of the Klein-Gordon equation with generalized hyperbolic potential in D-dimensions. J. Phys. Commun. 3, 095015 (2019).

15. B.I. Ita, H. Louis, O.U. Akakuru, N.A. Nzeata-Ibe, A.I. Ikeuba, T.O. Magu, P.I. Amos, C.O. Edet. Approximate solution to the Schrödinger equation with ManningRosen plus a class of Yukawa potential via WKBJ approximation method. Bulg. J. Phys. 45, 323 (2018).

16. C.O. Edet, U.S. Okorie, A.T. Ngiangia, A.N. Ikot. Bound state solutions of the Schrödinger equation for the modified 
Kratzer potential plus screened Coulomb potential. Indian. J. Phys. 94, 425 (2020).

17. S. Capozziello, G. Lambiase, G. Scarpetta. Generalized uncertainty principle from quantum geometry. Int. J. Theor. Phys. 39, 15 (2000).

18. A. Maireche. The Klein-Gordon equation with modified Coulomb plus inverse-square potential in the noncommutative three-dimensional space. Mod. Phys. Lett. A 35 (5), 2050015 (2020).

19. A. Maireche. The Klein-Gordon equation with modified Coulomb potential plus inverse-square-root potential in three-dimensional noncommutative space. Phys. J. 3, 186 (2019)

20. P.M. Ho, H.-C. Kao. Noncommutative quantum mechanics from noncommutative quantum field theory. Phys. Rev. Lett. 88 (15), 151602 (2002)

21. M. Darroodi, H. Mehraban, H. Hassanabadi. The KleinGordon equation with the Kratzer potential in the noncommutative space. Mod. Phys. Lett. A 33 (35), 1850203 (2018).

22. P. Gnatenko. Parameters of noncommutativity in Liealgebraic noncommutative space. Phys. Rev. D 99 (2), 026009-1 (2019).

23. P. Gnatenko, V.M. Tkachuk. Weak equivalence principle in noncommutative phase space and the parameters of noncommutativity. Phys. Lett. A 381 (31), 2463 (2017).

24. O. Bertolami, J.G. Rosa, C.M.L. De aragao, P. Castorina, D. Zappala. Scaling of varialbles and the relation between noncommutative parameters in noncommutative quantum mechanics. Mod. Phys. Lett. A 21 (10), 795 (2006)

25. A. Maireche. A recent study of excited energy levels of diatomics for modified more general exponential screened Coulomb potential: Extended quantum mechanics. J. Nano-Electron. Phys. 9 (3), 03021 (2017).

26. E.F. Djemaï, H. Smail. On quantum mechanics on noncommutative quantum phase space. Commun. Theor. Phys. (Beijing, China). 41 (6), 837 (2004).

27. Shi-Dong Liang, T. Harko. Toward an observable test of noncommutative quantum mechanics. Ukr. J. Phys. 64, 983 (2019).

28. Yi Yuan, Li Kang, Wang Jian-Hua, Chen Chi-Yi. Spin-1/2 relativistic particle in a magnetic field in NC phase space. Chinese Physics C 34 (5), 543 (2010).

29. O. Bertolami, P. Leal. Aspects of phase-space noncommutative quantum mechanics. Phys Lett. B 750, 6 (2015)

30. C. Bastos; O. Bertolami; N.C. Dias, J.N. Prata, WeylWigner formulation of noncommutative quantum mechanics. J. Mat. Phys. 49 (7), 072101 (2008).

31. J. Zhang. Fractional angular momentum in noncommutative spaces. Phys. Lett. B 584 (1-2), 204 (2004).

32. J. Gamboa, M. Loewe, J.C. Rojas. Noncommutative quantum mechanics. Phys. Rev. D 64, 067901 (2001)
33. M. Chaichian, Sheikh-Jabbari, A. Tureanu. Hydrogen atom spectrum and the Lamb shift in noncommutative QED. Phys. Rev. Lett. 86 (13), 2716 (2001).

34. A. Maireche. New relativistic atomic mass spectra of quark ( $\mathrm{u}, \mathrm{d}$ and $\mathrm{s}$ ) for extended modified Cornell potential in nano and Planck's scales. J. Nano-Electron. Phys. 8 (1), 010201 (2016).

35. A. Maireche. New bound state energies for spherical quantum dots in presence of a confining potential model at nano and Planck's scales. NanoWorld J. 1 (4), 122 (2016).

36. J. Wang, K. Li. The HMW effect in noncommutative quantum mechanics. J. Phys. A: Math. and Theor. 40 (9) $2197-$ 2202 (2007)

37. K. Li, J. Wang. The topological AC effect on non-commutative phase space. Europ. Phys. J. C 50 (4), 1007 (2007)

38. A. Maireche. A complete analytical solution of the mie-type potentials in non-commutative 3-dimensional spaces and phases symmetries. Afr. Rev. Phys. 11, 111 (2016).

39. A. Maireche. A new nonrelativistic investigation for the lowest excitations states of interactions in one-electron atoms, muonic, hadronic and Rydberg atoms with modified inverse power potential. Intern. Frontier Sci. Lett. 9, 33 (2016).

40. A. Maireche. New quantum atomic spectrum of Schrödinger equation with pseudo harmonic potential in both noncommutative three-dimensional spaces and phases. Lat. Am. J. Phys. Educ. 9 (1) 1301 (2015).

41. A. Maireche. New bound states for modified vibrationalrotational structure of supersingular plus Coulomb potential of the Schrödinger equation in one-electron atoms. Intern. Lett. Chem., Phys. Astronomy 73, 31 (2017).

42. A. Maireche. Extended of the Schrödinger equation with new Coulomb potentials plus linear and harmonic radial terms in the symmetries of noncommutative quantum mechanics. J. Nano- Electron. Phys. 10 (6), 06015-1 (2018)

43. A. Maireche. Investigations on the relativistic interactions in one-electron atoms with modified Yukawa potential for spin 1/2 particles. Intern. Frontier Sci. Lett. 11, 29 (2017)

44. R.L. Greene, C. Aldrich. Variational wave functions for a screened Coulomb potential. Phys. Rev. A 14 (6), 2363 (1976)

45. S.H. Dong, W.C. Qiang, G.H. Sun, V.B. Bezerra. Analytical approximations to the l-wave solutions of the Schrödinger equation with the Eckart potential. J. Phys. A: Math. Theor. 40 (34), 10535 (2007).

46. I.S. Gradshtein, I.M. Ryzhik. Table of Integrals, Series and Products. Edited by A. Jeffrey, D. Zwillinger (Rensselaer Polytechnic Institute, 2007). [ISBN-13: 978-0-12-373637-6, ISBN-10: 0-12-373637-4]

Received 21.08.19

ISSN 2071-0194. Ukr. J. Phys. 2020. Vol. 65, No. 11 
A. Мереш

НОВИЙ ПІДХІД ДО НАБЛИЖЕНОГО

АНАЛІТИЧНОГО РОЗВ'ЯЗКУ ТРИВИМІРНОГО

РІВНЯННЯ ШРЕДІНГЕРА ДЛЯ ВОДНЕПОДІБНИХ

І НЕЙТРАЛЬНИХ АТОМІВ У МОДЕЛІ

З УЗАГАЛЬНЕНИМ ПОТЕНЦАЛОМ ХЕЛЛМАНА

Р е 3 ю м е

В рамках нерелятивістичної некомутативної квантової механіки $з$ використанням схеми поліпшеного наближення для відцентрового члена для будь-яких $l$-станів в узагальненому методі зсуву Боппа і стандартної теорії збурень, ми отримали власні значення енергії для нещодавно запропонованої моделі узагальненого потенціалу Хеллмана (моделі УПХ) для воднеподібних і нейтральних атомів. Потен- ціал є суперпозицією кулонівського потенціалу тяжіння і потенціалу Юкави. Нові центральні члени виникають завдяки ефектам некомутативності простору і фази в моделі потенціалу Хеллмана. Отримані власні значення енергії виражаються через узагальнену гамма-функцію, дискретні атомні квантові числа $(j, n, l, s$ і $m)$, інфінітезимальні параметри $(a, b, \delta)$, породжені некомутативністю операторів (положення-положення і фаза-фаза), і залежать від розмірних параметрів $(\Theta, \bar{\theta})$ моделі УПХ в нерелятивістичному некомутативному тривимірному реальному фазовому просторі. Більше того, показано, що відповідний гамільтоніан із симетріями цього простору є сумою гамільтоніана моделі УПХ і двох операторів, один $з$ яких відповідає модифікованій спін-орбітальній взаємодії, а другий - це модифікований оператор Зеємана для воднеподібних і нейтральних атомів. 